\title{
Efficiency improvement in multi-sensor wireless network based estimation algorithms for distributed parameter systems with application at the heat transfer
}

\author{
Constantin Volosencu ${ }^{*}$ and Daniel-loan Curiac
}

\begin{abstract}
This paper gives a technical solution to improve the efficiency in multi-sensor wireless network based estimation for distributed parameter systems. A complex structure based on some estimation algorithms, with regression and autoregression, implemented using linear estimators, neural estimators and ANFIS estimators, is developed for this purpose. The three kinds of estimators are working with precision on different parts of the phenomenon characteristic. A comparative study of three methods - linear and nonlinear based on neural networks and adaptive neuro-fuzzy inference system - to implement these algorithms is made. The intelligent wireless sensor networks are taken in consideration as an efficient tool for measurement, data acquisition and communication. They are seen as a "distributed sensor", placed in the desired positions in the measuring field. The algorithms are based on regression using values from adjacent and also on auto-regression using past values from the same sensor. A modelling and simulation for a case study is presented. The quality of estimation is validated using a quadratic criterion. A practical implementation is made using virtual instrumentation. Applications of this complex estimation system are in fault detection and diagnosis of distributed parameter systems and discovery of malicious nodes in wireless sensor networks.
\end{abstract}

Keywords: Intelligent sensor networks, Distributed parameter systems, Estimation techniques, System monitoring, Virtual instrumentation

\section{Introduction}

The paper presents some theoretical and practical aspects of signal processing in the new and emerging technology of wireless sensor networks. The application is directed to the practicing engineers and also to the academic researchers. The problem covered in this paper is in the area of algorithms of multivariable estimation, architecture of system dedicated to process monitoring based on smart sensor and system implementation. The practical applications of this paper could be processes seen as distributed parameter systems. Advances in hardware and wireless network technologies have created smart, lowcost, low-power, multifunctional miniature sensor devices.

\footnotetext{
* Correspondence: constantin.volosencu@aut.upt.ro

Faculty of Automatics and Computers, Department of Automatics and Applied Informatics, "Politehnica" University of Timişoara, Bd. V. Parvan nr. 2,
} Timisoara 300223, Romania
The sensor number in a network is over hundreds or thousands of ad hoc tiny sensor nodes spread across different areas. Thus, the network actively participates in creating a smart environment. They are low cost and low energy devices, realized in nanotechnology. With them we may develop low cost wireless platforms, including integrated radio and microprocessors. These devices make up hundreds or thousands of ad hoc tiny sensor nodes spread across a geographical area. These sensor nodes collaborate among themselves to establish a smart sensing network. A sensor network can provide access to information anytime, anywhere by collecting, processing, analyzing and disseminating data. Wireless sensor networks are extremely distributed systems having a large number of independent and interconnected sensor nodes, with limited computational and communicative potential. The sensors are deployed for data acquisition purposes in a wide range

\section{穴}


of locations, sometimes in resource-limited and hostile environments such as disaster areas, seismic zones, ecological contamination sites and other different zones. This structure has the following characteristics: data processing is at the sensor level, data transmission is wireless, the sensing mechanism does not need more power supply. Sensor network applications include: environmental monitoring, civil infrastructure monitoring, shared resource utilization, tracking, perimeter protection and surveillance. Applications are in micro-climates, air quality, soil moisture, animal tracking, energy usage, office comfort, wireless thermostats, wireless light switches. In techniques they have applications such as data acquisition of physical and chemical properties, at various spatial and temporal scales, distributed parameter systems, for automatic identification, measurements over a long period of time. The modern sensors are smart, small, lightweight and portable devices, with a communication infrastructure intended to monitor and record specific parameters like temperature, humidity, pressure, wind direction and speed, illumination intensity, vibration intensity, sound intensity, power-line voltage, chemical concentrations and pollutant levels in diverse locations. The sensor networks are deployed for data acquisition purposes in a wide range of locations, in resource-limited and hostile environments such as disaster areas, seismic zones, contaminated ecological sites and so on. All these applications are distributed parameter systems. The nature of wireless sensor networks as smart and small distributed sensors could be of interest in a large class of smart and autonomous applications, capable to be implemented in multiple processes seen as largely distributed parameter processes. Also, the identification and malicious node detection in a distributed parameter system depends on sensor network interfacing with the real world. The sensors are adequate for autonomous operation in highly dynamic environments as distributed parameter systems. We may add sensors when they fail. They require distributed computation and communication protocols. They assure scalability, where the quality can be traded for system lifetime. They assure Internet connections via satellite. Based on the above consideration we may say: the intelligent sensor networks may be seen as a "distributed sensor" placed in the field of a distributed parameter system.

The paper presents the results of applied research and application of sensor networks as a new emerging type of "distributed sensor" for physical variables in engineering problems. These kinds of applications are adequate for estimation, monitoring, fault detection and diagnosis in distributed parameter systems. This application is framed in the field of industrial processes or environment systems which may be seen as distributed parameter systems. A distributed sensor network may be seen in this case as a "distributed" sensor placed into the field of a distributed parameter system. Examples of distributed parameter systems with large application in practice are: the process of heat conduction, applications related to the field of electricity, motion of fluids, the processes of cooling and drying, the phenomenon of diffusion and other applications are presented. The variables of these processes are: temperature, pressure, humidity, acceleration and so on. All of them may be measured with wireless sensor networks. The estimation made using sensor networks is useful for applications ranging from control systems, fault detection and diagnosis, signal processing to time-series analysis. In theory there are methods to estimate linear black box models and models of artificial intelligence for non-linear systems. Also, an important application may be the malicious node detection in wireless sensors, based on such estimation techniques. The data are input from sensors into automate systems using virtual instrumentation and adequate drivers.

The novelty value of this paper consists in applying some concepts such as sensor networks as a "distributed sensor", estimation techniques and virtual instrumentation to distributed parameter systems, with many applications in practice, such as system monitoring for fault detection and diagnosis, as it is illustrated in Figure 1.

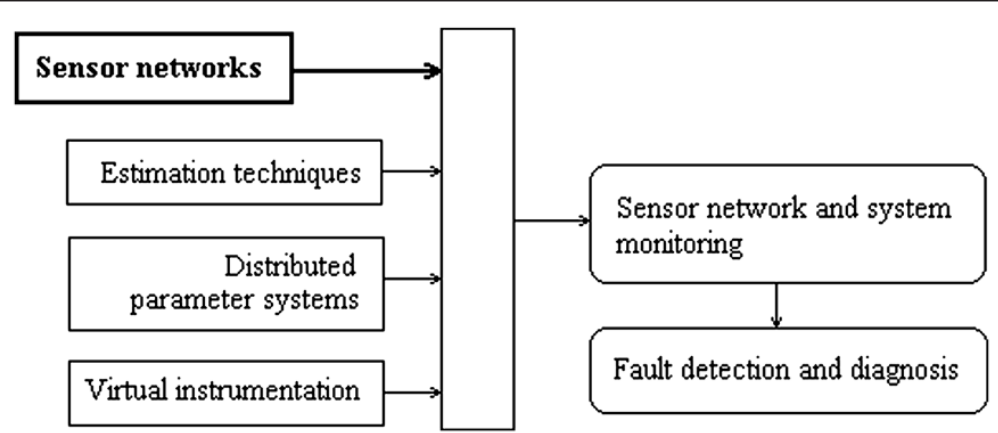

Figure 1 Domains of interest in practical application. 
Some simplifying methods for modelling these systems, that are trying to capture the distributed system dynamics through lumped parameter models, are used. The estimation equations were developed for estimator based on regression and auto-regression, with linear and non-linear models based on neural networks and adaptive neuro-fuzzy inference systems (ANFIS). A case study for the heat transfer, based on auto-regression estimator equation, is presented. Some modelling and simulation results are presented. The estimation results were compared based on the quadratic error criterion. For practical purposes a virtual instrument was built and tested for monitoring sensor networks, including the estimation. Based on the estimates, errors and faults in sensor network and of course in the field of distributed parameter systems, may be detected. A technical contribution based on multivariable estimation techniques, distributed parameter system theory and virtual instrumentation is presented with consistency. Starting from three standard methods to implement the novel estimation algorithms based on sensor networks for distributed parameter systems, in the discretized version of them, we are showing what is the better method to increase the quality of estimation, related to the complexity of implementation. The role of the sensor network is a major one, because it is seen as a distributed sensor placed in the field. All the critical aspects which are arising in identification of distributed parameter systems from large data are taken into consideration: the structure of the estimator is determined, an adequate variable selection is made and last but not least the distributed computation is solved using the distributed intelligent sensor placed into a network with a collecting base station. There is a substantial and structured overview of references in the chapter of related work, focused on the problem. The paper starts from the classical model of the parabolic distributed parameter systems, which are presented in an implicit form, then in an explicit form.

The main contribution of this paper is a complex estimation structure, resulted using combined two estimation algorithms, with regression and auto-regression, developed in other papers, implemented using three efficient estimation methods: one linear and two non-linear - with neural networks and ANFIS. This complex system is used to estimate the value of the sensor at the moment $t+1$, based on the past values of the same sensor and also based on the current values of the adjacent sensors. One of the estimation algorithms is using the values of the adjacent sensors and the other algorithm is using the antecedent values of the same sensor. A discrete approximation of parabolic models and the two estimation algorithms presented in the paper have already tested on different case studies, and the results were been published by the authors in references [1,2,3,4]. A short consideration is made on this knowledge, with simple equations, offered to understand how the complex estimator works. From the explicit form of the equation, which is a more general form of the distributed parameter system, the possible order of the estimator is determined, the variables are selected, and two computation algorithms are presented. Following these considerations the model structure imposed by the distributed parameter system dynamics is modelled into the estimation algorithms. The meshes in the distributed system are a good indication how to place the sensors in the field. The neural estimator was previously tested on some case studies, and the results were presented in [5]. The ANFIS estimator was tested on some case studies, and the results were presented in [3]. The ANFIS estimator with the estimation algorithms were tested on a practical application in environmental monitoring and the results were presented in [4].

The intelligent sensor network with its base station, the PC drivers and the virtual instrumentation solved the problem of the associated constrains - communication, computation and so on. The practical contribution of this paper is that it presents how to improve the estimation quality in the case of an estimation using sensor networks, in a real time application, for fault detection and diagnosis.

\section{Related work}

Since for distributed parameter systems it is impossible to observe their states over the entire spatial domain, a possible solution is to locate discrete sensors to estimate the unknown system parameters as accurately as possible. There is recent original work on optimal sensor placement strategies for parameter identification in dynamic distributed systems modeled by partial differential equations. The development of new techniques and algorithms or adopting methods, which have been successful in the field of optimal control and optimum experimental design, is reported in different papers. Advances in scientific computation and developments in spatial sensor technology have enhanced the ability to develop modelling strategies and experimental techniques for the study of the space-temporal response of distributed non-linear systems. Robust implementations of distributed system identification algorithms based on detailed space and time experimental data have an important role in practical applications. Wireless automation is today an emerging topic.

In many sensor networks applications, sensors collect correlated measurements of a physical field, in a building or in a data center [6-10]. However, the locations of the sensors are usually inconsistent with the application requirements. In the papers $[11,12]$ the problem of estimating the field at arbitrary positions of interest, where there are possibly no sensors, from the irregularly placed sensors is considered. The sensor network on a graph is mapped, and 
by introducing the concepts of interconnection matrices, system digraphs, and cut point sets, real-time field estimation algorithms are derived. Simulations and real world experiments on temperature estimation are conducted.

A strategy by which sensor nodes detect and estimate non-localized phenomena such as boundaries and edges (e.g., temperature gradients, variations in illumination or contamination levels) can be found in study [13]. A general class of boundaries, with mild regularity assumptions, is considered, and a theory on the achievable performance of sensor network based boundary estimation is established. A hierarchical boundary estimation algorithm is proposed that achieves a near-optimal balance between mean-squared error and energy consumption.

In the paper [14] the state of the art algorithms for consensus-based distributed estimation using ad hoc wireless sensor networks, where sensors communicate over single-hop noisy links, are presented. Basic estimation criteria such as least-squares, maximum-likelihood and others are reformulated in a novel framework, amenable to distributed solutions. The framework encompasses adaptive filtering and smoothing of non-stationary signals through distributed LMS and Kalman filtering.

The functions of different wireless technologies are described in $[15,16]$. In the survey paper for system identification [17] it is shown that the sensor networks represent "an open area in system identification, being a rapidly evolving technology to collect information with many spatial distributed, autonomous devices. They have an interesting potential for industrial monitoring purposes and add to the richness of information for model development." The development of wireless sensor allows the application of many methods and algorithms for identification of systems, with a high efficiency in the particular case of the distributed parameter systems. The main principles consist in the fact that in this kind of identification the sensor network may be seen as a "distributed sensor" placed into a field, which is a distributed parameter system, allowing measurement in well-chosen points of an infinite variable system. In the emerging area of research in wireless sensor network the applications paper [18] presents the design of a wireless network sensor, based on a database, which archives the data reported by distributed sensors, as well as the implemented support for queries and data presentation. In the paper [19] an architecture of a set of mobile sensors is developed were the sensors collaborate with the stationary sensors in order to detect an event, for an accurate view of the state of the environment. The special issue [20] brings together contributions from signal processing, communications and new algorithm design methodologies of cooperative localization systems. An application of wireless sensor networks for RF-based indoor localization is presented in [21]. In the special issue [22] the state of the art and emerging distributed signal processing techniques in wireless sensor networks, are presented, estimation being one of them. In the special issue [23] the recent interest in developing sensor networks for different sensible applications, for area monitoring, including detecting, identifying, locating and tracking the emission signals of interest is emphasized. The special issue [24] deals with various signal processing aspects of sensor networking, which are significant for providing measurements of the physical phenomenon around, for understanding and using this information in a wide range of potential applications including environmental monitoring, health care monitoring, harsh field surveillance and reconnaissance, modern manufacturing, condition based maintenance of complex systems, and so forth.

For sensor network monitoring there are different technical solutions. In the paper [25] a software environment for monitoring and controlling sensor network via a web interface is presented.

Some recent applications of sensor networks in which distributed parameter process monitoring is of interest are presented as follows. Thus, a possible application of sensor networks, in a field with distributed points, is the tracking problem of a dynamic object movement between these distributed points on the field. The paper [26] presents a technical solution based on acoustic and visual sensors. Another solution for real time tracking in wireless sensor networks is presented in the paper [27]. The usage of estimation for monitoring service attacks in sensor networks is an important application. A technical solution for solving this problem using algorithms for filtering bogus signals from different sensors from the field is presented in the paper [28]. An example of industrial process application in manufacturing plasma treatment for polymers is presented in the paper [29].

Some papers in which the sensor networks are used for estimation purposes are [30], where a graph approach is presented, and [31], where for estimation reduced order physical models are used.

Paper [32] is focused on distributed state estimation using a sensor network for monitoring a linear system. A moving horizon approach is proposed, where each sensor has to solve a quadratic programming problem at each instant. Paper [12] deals with the problem of estimating the state of a discrete-time linear stochastic dynamic system on the basis of the data collected from multiple sensors. In the paper [33] each node of a sensor network is collecting noisy linear measurements of some unknown parameter. A consensual diffusion scheme is studied on bi-directional neighbour nodes. Each node is computing a convergent estimate of the unknown parameter. The theoretical study considers networks with dynamically changing communication topology. In the paper [13] an algorithm for the identification of 
distributed systems by large-scale collaborative sensor networks is initiated, based on the distributed KarhunenLoève transformation. The reduction of the distortions at the nodes and the low computational complexity of the fusion algorithm at the fusion centre are taken into consideration. The algorithm is applied on a system described by a partial differential equation in 2-D domain. A state estimation problem is considered in the paper [34], based on a Kalman filter, in which two communication schemes are producing estimates at the fusion centre. Some simulations are provided, under various circumstances. The paper [35] presents an application of sensor networks for distributed $\mathrm{H}_{\infty}$ consensus filtering with multiple missing measurements. The identification of non-linear systems continues to be a contemporary problem, trying to be solved using different methods, for example in [36] for unknown nonlinear system, given the distribution knowledge of the system inputs. Artificial neural networks have seen significant applications over the last decades in the area of system identification, as a flexible way to give hypersurfaces for regression, being very effective for solving a large number of non-linear estimation problems [17]. The paper [37] treats an application of partial differential equations, considering that neural networks can approximate a large set of continuous functions defined on a compact set to an arbitrary accuracy. The paper [9] presents an application of unsupervised regression at non-linear system identification. An approximation algorithm for optimising a cost function is developed, as manifold learning using low dimensional coordinates. ANFIS is a well-known method for development self-organizing neuro-fuzzy systems with many applications in practice. After years of trying to find new algorithms, ANFIS is still in use, and recent applications were reported such as [38] or an application of ANFIS at sensor data processing [39] or [11]. The paper [26] presents some aspects related to the estimation of average air temperature in the built environment by using integer neural networks, ANFIS and inferential sensor models. The paper compares the results of these models, presenting their advantages and disadvantages. Time series predictions based on ANFIS are presented in the literature, for example [40]. Applications of fault detection and diagnosis in distributed parameter systems are presented as follows. In the paper [14] the problem of fault detection in distributed parameter systems is formulated as maximizing the power of a parameter hypothesis testing whether the system parameters have nominal values or not. The sensor locations are given a priori. A gradient projection algorithm is proposed to perform the search for the optimal solution. The approach is illustrated by a numerical example for a two-dimensional diffusion process. The paper [41] considers the problem of designing fault diagnosis algorithms for dynamic systems using sensor networks. A network of distributed estimation agents is designed, with local Kalman filter embedded into each sensor. The diagnosis decision is performed by a distributed hypothesis testing method that relies on a belief consensus algorithm. Simulation results are provided to demonstrate the effectiveness of the proposed architecture and algorithm.

\section{General mathematical model of the parabolic distributed parameter system with application at the heat transfer}

The distributed parameter systems have general mathematical models in continuous time and space as partial differential equation, of parabolic form, as:

$$
\frac{\partial \theta}{\partial t}=c_{1} \nabla\left(c_{2} \nabla \theta\right)+c_{3} \theta+Q
$$

where the variables $\theta(\zeta, t)$ depend on time $t \geq 0$ and on space $\zeta \in V$, where $\zeta$ is $x$ for one axis and $(x, y)$ for two axis, $c_{1}, c_{2}$ and $c_{3}$ are coefficients, which could be also time variant and $Q(\zeta, t)$ is an exterior excitation, variable on time and space. So, in the general case, an implicit equation may be written:

$$
f\left(\frac{\partial \theta}{\partial t}, \frac{\partial \theta}{\partial \zeta}, \frac{\partial^{2} \theta}{\partial \zeta^{2}}, \ldots\right)=0
$$

For the partial differential equation (1) some boundary conditions may be imposed to establish a solution. So, when the variable value of the boundary is specified there are Dirichlet conditions:

$$
c_{4} \theta=q
$$

and, when the variable flux and transfer coefficient are specified there are Neumann conditions:

$$
c_{5} \nabla \theta+c_{6} \theta=0
$$

In the practical application case studies limits and initial conditions of the equation (1) are imposed:

$$
\begin{aligned}
& \theta(0, t)=\theta_{\zeta 0}, t \in[0, T], \theta(\zeta, 0)=0, \zeta \in[0, l], \\
& \theta(l, t)=\theta_{\zeta l}, t \in[0, T]
\end{aligned}
$$

A system with finite differences may be associated with the equation (1). For this purpose the space $S$ is divided into small pieces of dimension $l_{\mathrm{p}}$ :

$$
l_{p}=l / n
$$

In each small piece $S_{\mathrm{pi}}, i=1, \ldots, n$ of the space $\mathrm{S}$ the variable $\theta$ could be measured at each moment $t_{\mathrm{k}}$, using an intelligent sensor from the sensor network, in a characteristic point $\mathrm{P}_{\mathrm{i}}\left(\zeta_{\mathrm{i}}\right)$, of the coordinate $\zeta_{\mathrm{i}}$. Let it be $\theta_{\mathrm{i}}^{\mathrm{k}}$ the variable value in the point $\mathrm{P}_{\mathrm{i}}\left(\zeta_{\mathrm{i}}\right)$ at the moment $t_{\mathrm{k}}$. It is a well known method to approximate the derivatives of a variable with small variations. In the equation with partial derivatives there are derivatives of first order, in 
time, and derivatives of first and second order in space. So, theoretically, we may approximate the variable derivatives in time with small variations in time, with the following relation:

$$
\frac{\partial \theta}{\partial t}=\frac{\theta_{i}^{k+1}-\theta_{i}^{k}}{t_{k+1}-t_{k}}
$$

The first and the second derivatives in space may be approximated with small variations in space to obtain the following relations. For the $x$-axis we may write the following equations:

$$
\frac{\partial \theta}{\partial x}=\frac{\theta_{i}^{k}-\theta_{i-1}^{k}}{l_{p}}, \frac{\partial^{2} \theta}{\partial x^{2}}=\frac{\theta_{i+1}^{k}-2 \theta_{i}^{k}+\theta_{i-1}^{k}}{l_{p}^{2}}
$$

The same equations may be written also for the $y$ axis. Of course, an equation with variables written in vectors could be written. We may consider the variable is measured as sample $\theta_{i}^{k}=\theta\left(\zeta_{i}, t_{k}\right), \zeta_{i} \in V$, at equal time intervals with the value:

$$
h=t_{k+1}-t_{k}
$$

called sample period, in a sampling procedure, with a digital equipment, at the sample time moments $t_{\mathrm{k}}=k h$. For the parabolic equation a linear approximate system of derivative equations of first degree may be used:

$$
\frac{d \Psi}{d t}=A \Psi+B Q
$$

where, this time, $\psi$ is a vector containing the values of the variable $\theta(\zeta, t)$ in different points of the space and at different moments in time.

Combining the equations $(7,8)$ in the equation $(1)$ a system of equations with differences results for the parabolic equation $[1,3,4]$ :

$$
f_{p}\left(\theta_{i}^{k}, \theta_{i-1}^{k}, \theta_{i}^{k+1}, \theta_{i+1}^{k}\right)=0
$$

Several estimation algorithms may be developed as follows, based on the discrete models of the partial derivative equations, taking account of the equations (11).

\section{Multisensor network based estimation algorithms and monitoring method \\ 4.1 Estimation algorithms}

Analysing the form of the equation (11) we may see a number $n=4$ of variables involved: $\theta_{i}^{k}, \theta_{i-1}^{k}, \theta_{i}^{k+1}, \theta_{i+1}^{k}$, which are the measured variables from the sensors placed in 3 adjacent points $\mathrm{P}_{\mathrm{i}-1}\left(\zeta_{\mathrm{i}-1}\right), \mathrm{P}_{\mathrm{i}}\left(\zeta_{\mathrm{i}}\right)$ and $\mathrm{P}_{\mathrm{i}+1}\left(\zeta_{\mathrm{i}}\right)$, $i=1, \ldots, n$ of the space $\mathrm{S}$, at two moments: $t_{\mathrm{k}}$ and $t_{\mathrm{k}+1}$. Based on the structure of the equation (11) we may develop estimation algorithms using the values measured at 3 adjacent sensors: $\theta_{i-1}^{k}, \theta_{i+1}^{k}, \theta_{i}^{k}$ or using the antecedent values of the same sensor: $\theta_{i}^{k}, \theta_{i}^{k-1}, \theta_{i}^{k-2}, \theta_{i}^{k-3}$.
These novel estimation algorithms, which are developed for multisensor networks are presented as follows $[1,3,4]$.

Estimation algorithm 1. It estimates the value of the variable $\theta_{i}^{k+1}$ at the moment $t_{\mathrm{k}+1}$, measuring the values of the variables $\theta_{i-1}^{k}, \theta_{i+1}^{k}, \theta_{i}^{k}$ at the antecedent moment $t_{\mathrm{k}}$ :

$$
\theta_{i}^{k+1}=f_{1}\left(\theta_{i-1}^{k}, \theta_{i+1}^{k}, \theta_{i}^{k}\right)
$$

This is a multivariable estimation algorithm, based on adjacent points in space (nodes or sensors).

Estimation algorithm 2. It estimates the value of the variable $\theta_{i}^{k+1}$ at the moment $t_{\mathrm{k}+1}$, measuring the values of the same variable $\theta_{i}^{k}, \theta_{i}^{k-1}, \theta_{i}^{k-2}, \theta_{i}^{k-3}$, but at four anterior moments $t_{\mathrm{k}}, t_{\mathrm{k}-1,}, t_{\mathrm{k}-2}$ and $t_{\mathrm{k}-3}$.

$$
\theta_{i}^{k+1}=f_{2}\left(\theta_{i}^{k}, \theta_{i}^{k-1}, \theta_{i}^{k-2}, \theta_{i}^{k-3}\right)
$$

The estimator could be a linear or a non-linear one, described by the function $y=f\left(u_{1}, u_{2}, \ldots, u_{\mathrm{n}}\right)$, using different estimator implementation methods. In this paper three estimator implementation methods are used and compared: a linear estimator, an estimator based on a feedforward neural network with continuous values and an estimator based on an ANFIS. The number of inputs depends on the estimation algorithm, on the specific position in space of the measuring points, and on the conditions of determination.

\subsection{Monitoring method}

The defined distributed system in the practical application is the process of heat distribution, in the real world. The following method is according to the objectives of monitoring of this real process. These systems have known mathematical model as a parabolic partial differential equation as a primary model from physics, with well-defined boundary and initial conditions for the system in practice. These represent the basic knowledge for a reference model from real data observation. The primary physical model must be discretized, to obtain a mathematical model as a MIMO state-space model. The unstructured meshes may be generated.

In the field of the distributed parameter system several sensors are placed, as it is presented in Figure 2.

For each point $\mathrm{P}_{\mathrm{A}}$ there is a sensor $\mathrm{S}_{\mathrm{A}}$ and around it there are other 4 adjacent points $\mathrm{P}_{\mathrm{adj} 1}, \mathrm{P}_{\mathrm{adj} 2}, \mathrm{P}_{\mathrm{adj} 3}$ and $\mathrm{P}_{\text {adj4 }}$ in which there are placed 4 sensors $\mathrm{S}_{\text {adj1 }}, \mathrm{S}_{\text {adj2 }}, \mathrm{S}_{\text {adj3 }}$ and $S_{\text {adj4, }}$, like in Figure 3.

The sensors must be placed in the field according to the meshes structured under the form of nodes and triangles. The constructive and functional representation of a sensor network used in practice is presented in Figure 4. 


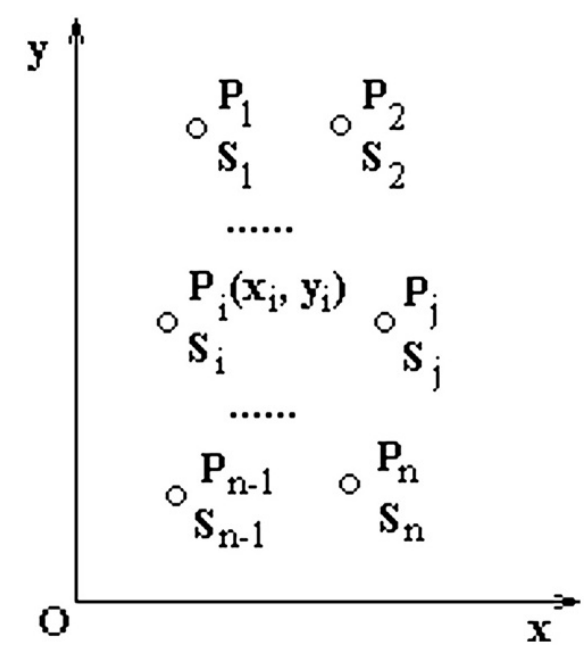

Figure 2 Sensor placed in the field.

The sensor network, made by hundreds of ad-hoc tiny sensor nodes is spread across the space. Sensor nodes collaborate among themselves, and the sensor network provides information anytime, by collecting, processing, analyzing and disseminating temperature measured data. New nodes are automatically detected and incorporated. The number and the place point of the de sensor nodes may be discussed according to the desired accuracy of estimation. A scenario for practical applications could be chosen and simulated. The simulation and the practical measurements are producing transient regime characteristics. Those transient characteristics are due to the system dynamics in a training process. In a steady state we cannot obtain an estimator model. On these transient characteristics, seen as times series, the estimation algorithms may be applied. Some estimators may be used to

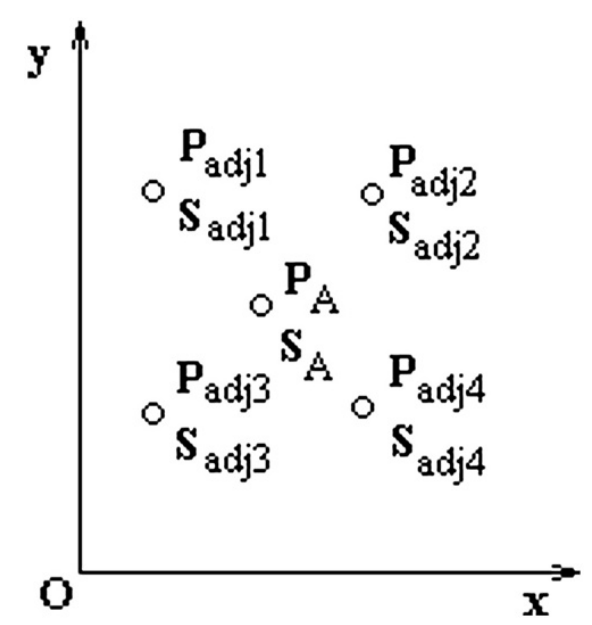

Figure 3 Adjacent points and sensors.

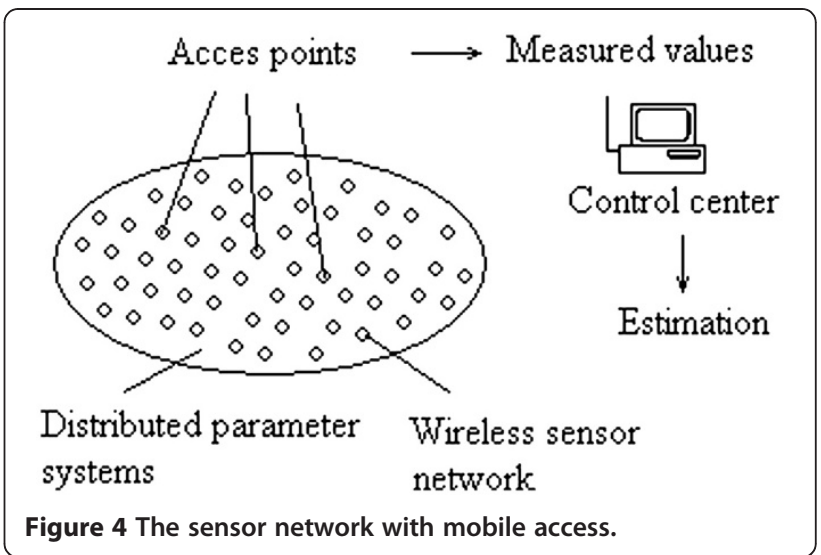

implement the estimation algorithms. With these algorithms future states of the process may be estimated. Possible faults in the system are chosen and strategies for detection may be developed, to identify and to diagnose them, based on the state estimation. In practice applying the method presumes the following steps: -placing a sensor network in the field of the distributed parameter system; -acquiring data, in time, from the sensor nodes, for the system variables; -using measured data to determine an estimation model; -using measured data to estimate the future values of the system variables; -imposing an error threshold for the system variables; -comparing the measured data with the estimated values; -if the determined error is greater, then a threshold default occurs; -diagnosing the default, based on estimated data, determining its place in the sensor network and in the distributed parameter system field.

Based on this placement of sensors some estimation algorithms may be developed. For only one sensor $S_{A}$ an estimation algorithm based on auto-regression of the values acquired from this sensor at several antecedent moments may be used. Using the 4 adjacent sensors an estimation algorithm with regression of the values from these sensors at the same moment may be used. To implement these two types of algorithms several estimation functions may be used. One of them may be a linear one. Other two may be non-linear, for example a neural one and an ANFIS one. According to these assumptions, a combination of six algorithms results. The comparison of the algorithms and their results is made by using a quadratic quality criterion. For each algorithm a quality criterion is calculated at each estimation. The decision related to the estimation efficiency is taken into a decision block, using several decision obtained by reasoning. And the best estimate is provided at the output of this strategy for efficiency improvement.

The estimators are tuned or trained using some study cases adequately chosen for the distributed parameter system. 
The structures of the estimators for a study case will be presented in the following part of the paper.

Several quadratic error criteria may be used.

\subsection{The complex estimation structure}

To increase the efficiency of estimation in a multisensor monitoring system dedicated to a distributed parameter system the structure from Figure 5 is proposed.

The reason for using three types of estimators is as follows. The process to be estimated has characteristics which are linear, non-linear of a low degree and high non-linear in some parts. The estimators are chosen for each of these three types of characteristics to give the best estimate, with the smallest error: linear estimator, to estimate the linear parts, neural estimator, to estimate parts with a low non-linearity level and ANFIS estimator, to estimate the high level non-linear parts.

\section{Methods to implement the estimation algorithms}

\subsection{Linear estimator}

When a linear estimation method is used for implementation the estimator has the following general equation:

$$
y=a_{1} u_{1}+a_{2} u_{2}+a_{3} u_{3}+a_{4} u_{4}
$$

where $y$ is the estimate, obtained by using four input variables $u_{1}, u_{2}, u_{3}$ and $u_{4}$ and $a_{\mathrm{i}}$ are constant coefficients, obtained by using a least square method. To design the estimators of the $i$ sensor, for $i=1, \ldots, m$ sensors and points in space, a set of $N$ measured variables, from the sensor networks are required:

$$
x_{i}\left(t_{k}\right), i=1, \ldots m, k=1, \ldots, N
$$

An error is imposed:

$$
\begin{aligned}
e_{i, k} & =x_{i}\left(t_{k}\right)-x_{i}\left(t_{k}\right), i=1, \ldots, m, k \\
& =1, \ldots, N
\end{aligned}
$$

And a quadratic quality criterion, too:

$$
J_{i}=\frac{1}{2} \sum_{j=1}^{N} e_{i, k}^{2}, \quad i=1, \ldots, m
$$

The estimator equation is:

$$
\begin{aligned}
& x_{i}\left(t_{k+1}\right)=a_{i, 0} x_{i}\left(t_{k}\right)+a_{i, 1} x_{i}\left(t_{k-1}\right)+a_{i, 2} x_{i}\left(t_{k-2}\right)+ \\
& +a_{i, 3} x_{i}\left(t_{k-3}\right), i=1, \ldots, m, k=3, \ldots, N-1
\end{aligned}
$$

The estimator coefficients are obtained using the least square method:

$$
\frac{\partial J}{\partial a_{i}}=0, i=0,1,2,3
$$

Some examples of the linear estimator coefficients obtained for the case study are presented in the paper.

\subsection{Neural estimator}

The neural network used for estimation is a feedforward neural network, with continuous values [2]. It has 4 inputs, which may be, for the auto-regression algorithm the sensor values at 4 antecedent moments $u_{1}=\mathrm{x}_{\mathrm{A}}(\mathrm{t}-1)$, $u_{2}=\mathrm{x}_{\mathrm{A}}(\mathrm{t}-2), u_{3}=\mathrm{x}_{\mathrm{A}}(\mathrm{t}-3)$ and $u_{4}=\mathrm{x}_{\mathrm{A}}(\mathrm{t}-4)$, or for the regression algorithm four values of adjacent sensors at the same time moment $u_{1}=\mathrm{x}_{\text {adj } 1}(\mathrm{t}), u_{2}=\mathrm{x}_{\mathrm{adj} 2}(\mathrm{t}), u_{3}=\mathrm{x}_{\text {adj } 3}(\mathrm{t})$ and $u_{4}=\mathrm{x}_{\mathrm{adj} 4}(\mathrm{t})$. Its structure is presented in Figure 6.

According to Kolmogorov's theorem two hidden layers of neurons with biases are used, to obtain a reduced error of approximation of the estimate $f$. The input layer

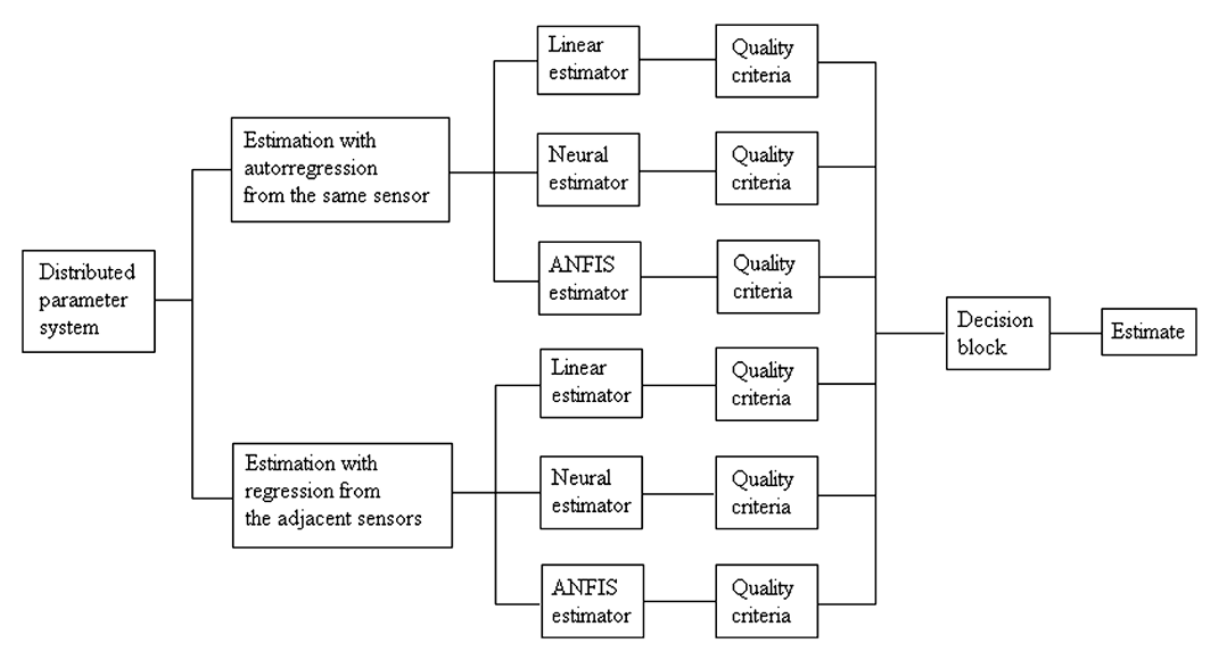

Figure 5 The block diagram of the estimation process. 


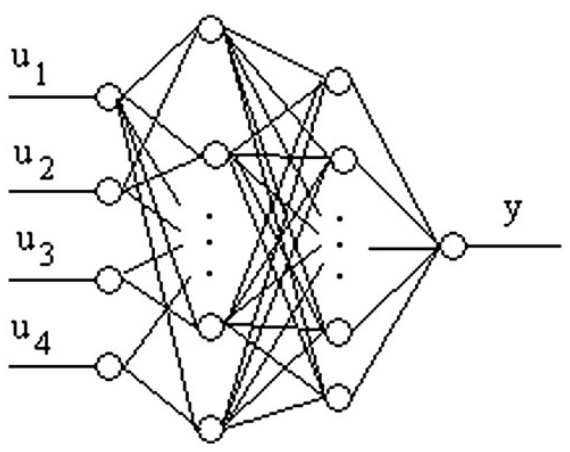

Figure 6 Neural network structure.

has 4 neurons, for the previous values of the measured temperatures. The output layer has one neuron for the estimated temperature. The first and the second hidden layers have a reduced number of neurons, 32 and 16 neurons, respectively. These numbers resulted after some iterative training. The activation functions of the neural network are the hyperbolic tangent function for the hidden layers and the first-order linear function for the output layer. The method chosen for training was the Levenberg-Marquardt method, using the set of $N$ measured values as a training set, in a different scenario of transient characteristics.

\subsection{ANFIS estimator}

The ANFIS estimator is a non-linear one, described by a function $y=f\left(u_{1}, u_{2}, u_{3}, u_{4}\right)$, using the adaptive-networkbased fuzzy inference [3,4]. Its general structure is presented in Figure 7.

A short description of the ANFIS and its function approximation property is provided as follows. For the estimation algorithms there are 4 inputs, because of the first order derivation in time of the parabolic model. The ANFIS procedure may use a hybrid-learning algorithm to identify the membership function parameters of single-output, the Sugeno type fuzzy inference system. A combination of least-squares and backpropagation gradient descent methods may be used for training membership function parameters, modelling a given set of input/output data. In the inference method and may be implemented with product or minimum, or may be implemented with maximum or summation, implication may be implemented with product or minimum and aggregation may be implemented with maximum or arithmetic media. The first layer is the input layer. The second layer represents the input membership or fuzzification layer. The neurons represent

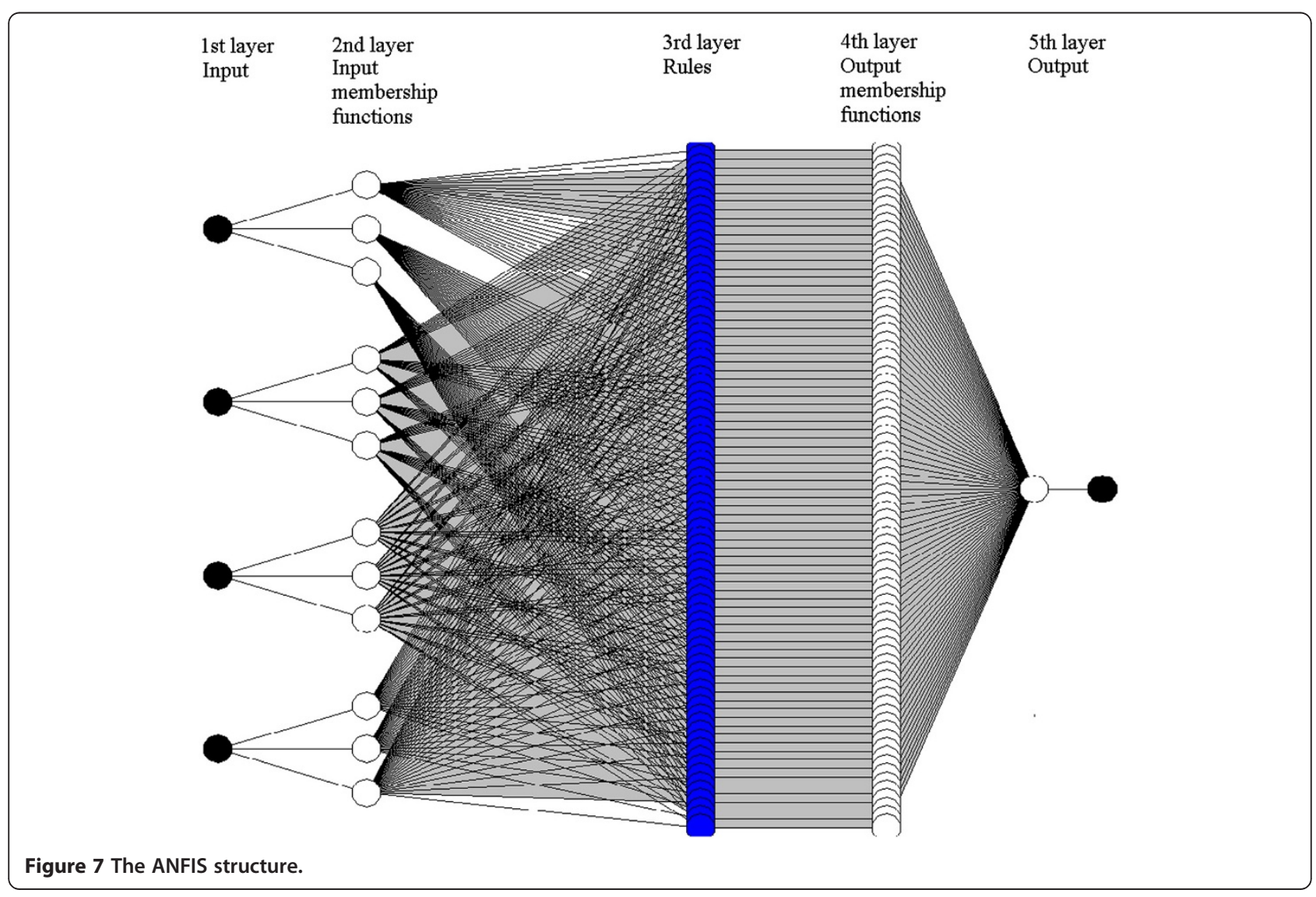




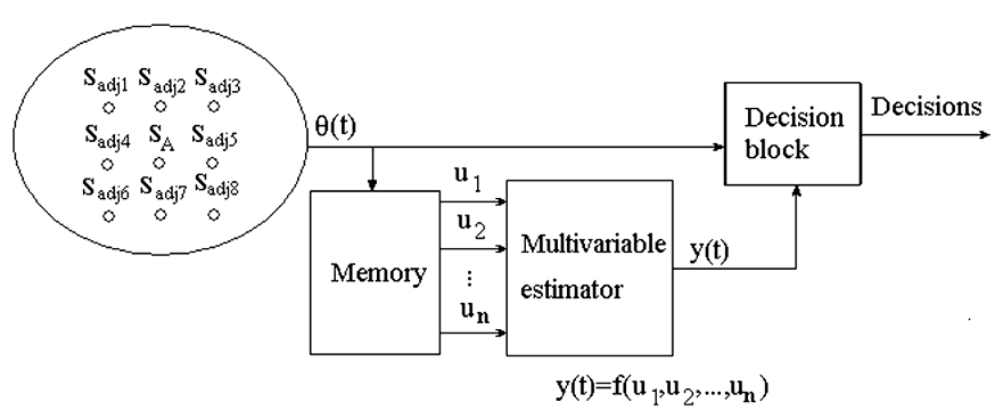

Figure 8 Estimation and detection structure.

fuzzy sets used in the antecedents of fuzzy rules and determine the membership degree of the input. The activation function represents the membership function. The $3^{\text {rd }}$ layer represents the fuzzy rule base. Each neuron corresponds to a single fuzzy rule from the rule base. The inference is in this case the sum-prod inference method, the conjunction of the rule antecedents being made with product. The weights of the $3^{\text {rd }}$ and $4^{\text {th }}$ layers are the normalized degree of confidence of the corresponding fuzzy rules. These weights are obtained by training in the learning process. The $4^{\text {th }}$ layer represents the output membership function. The activation function is the output membership function. The $5^{\text {th }}$ layer represents the defuzzification layer, with single output, and the defuzzification method is the centre of gravity. The training set is the measured $N$ values obtained from the sensor network.

\section{Methodology validation for the estimation algorithms in application}

\subsection{Estimation and detection structure}

The estimation model describes the evolution of a variable measured over the same sample period as a function of past evolutions. This kind of system evolves due to its "memory", generating internal dynamics. The estimation model definition is:

$$
y(t)=f\left(u_{1}(t), \ldots, u_{4}(t)\right)
$$

where $u(\mathrm{t})$ is a vector of the series under investigation.
In our case, it is the series of values measured by the sensors from the network:

$$
u=\left[\begin{array}{llll}
u_{1} & u_{2} & u_{3} & u_{4}
\end{array}\right]^{T}
$$

and $f$ is the estimation function of regression, 4 is the order of the regression. By convention all the components $u_{1}(\mathrm{t}), \ldots, u_{4}(\mathrm{t})$ of the multivariable time series $u(\mathrm{t})$ are assumed to be zero mean. The function $f$ may be estimated in case the time series $x_{\mathrm{i}}(\mathrm{t}), x_{\mathrm{i}}(\mathrm{t}-1), \ldots, x_{\mathrm{i}}(\mathrm{t}-\mathrm{n})$ is known (recursive parameter estimation), or may predict future value in case the function $f$ and past values $x_{\mathrm{i}}$ $(\mathrm{t}-1), \ldots, x_{\mathrm{i}}(\mathrm{t}-\mathrm{n})$ are known (auto-regression prediction). The method uses the time series of measured data provided by each sensor and relies on an auto-regressive multivariable predictor placed in base stations as it is presented in Figure 8.

The principle of the estimation is the following: the sensor nodes will be identified by comparing their output values $\theta(t)$ with the values $y(t)$ predicted using past/present values provided by the same sensors. After this initialization, at every instant moment $t$ the estimated values are computed relying only on past values $\theta_{\mathrm{A}}(\mathrm{t}-1), \ldots, \theta_{\mathrm{A}}(0)$ and both parameter estimation and prediction are used. The parameters of the function $f$ are estimated for each of three estimator methods: linear, neural and ANFIS, using a set of $N$ measured values. After that, the present values $\theta_{A}(t)$ measured by the sensor nodes may be compared with

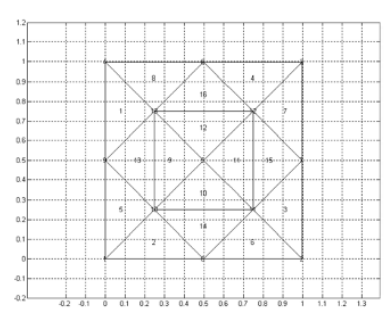

a)

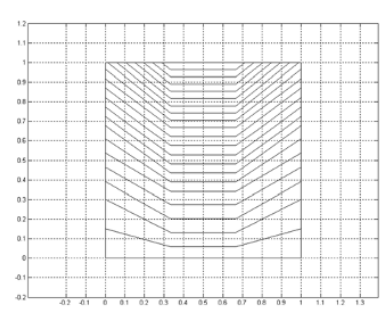

b)

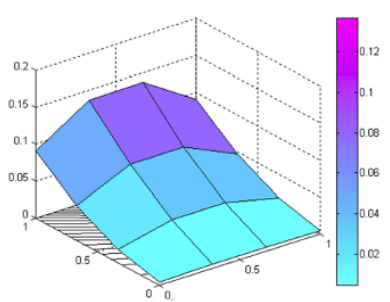

c)

Figure 9 Characteristics of a reduced number of sensors. a) Meshes and nodes $\mathbf{b})$ temperature isotherms, and $\mathbf{c}$ ) temperature in 3D. 


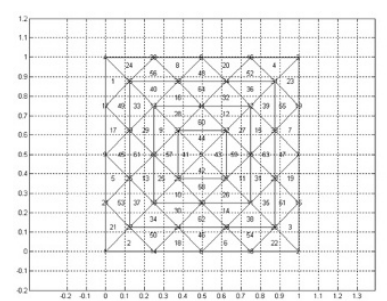

a)

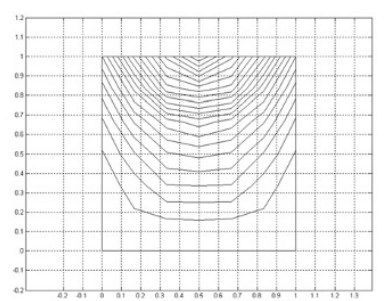

b)

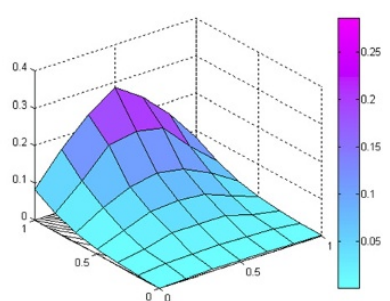

c)

Figure $\mathbf{1 0}$ Characteristics of an average number of sensors. a) Meshes and nodes $\mathbf{b}$ ) temperature isotherms, and $\mathbf{c}$ ) temperature in 3D.

their estimated values $y(t)$ by computing the errors $(\mathrm{A}=i)$ :

$$
e_{A}(t)=\left|\theta_{A}(t)-y(t)\right|
$$

If these errors are higher than the thresholds $\varepsilon_{A}$ at the sensor measuring point a fault occurs. Here, based on a database containing the known models, on a knowledge-based system we may see the case as a multi-agent system, which can provide criticism, learning and changes, taking decision based on node analysis from network topology. Two parameters can influence the decision: the type of the distributed parameter system, which is offering the data measured by sensors and the computing limitations. Because both of them are known a priori an off-line methodology is proposed. A realistic value of the recursive order was chosen to be 4 , according to the number of inputs from the estimation equations.

\subsection{Case study}

A basic case study consisting in a heat distribution flux through a plane square surface of dimensions $l=1$, with Dirichlet boundary conditions at constant temperature on three margins is presented:

$$
h_{\theta} \theta=r
$$

with $r=0$, and a Neumann boundary condition as a flux temperature from a source

$$
n k \nabla \theta+q \theta=g
$$

where $q$ is the heat transfer coefficient $q=0, g=0, h \theta=1$.

The heat equation, of a parabolic type, is

$$
\rho C \frac{\partial \theta}{\partial t}=\nabla(k \nabla \theta)+Q+h_{\theta}\left(\theta_{e x t}-\theta\right)
$$

where $\rho$ is the density of the medium, $C$ is the thermal (heat) capacity, $k$ is the thermal conductivity, coefficient of heat conduction, $Q$ is the heat source, $h \theta$ is the convective heat transfer coefficient, $\theta_{\text {ext }}$ is the external temperature. Relative values are chosen for the equation parameters: $\rho C=1, Q=10, k=1$.

The positions of the meshes and nodes, the temperature variation as isotherms and variation in $3 \mathrm{D}$ are presented in Figures 9, 10 and 11, for three analysis cases.

An analysis is done related to the number and position of the sensors in the space to obtain more accurate measurements. An optimised solution of over 150 meshes and nodes to place sensors in space is presented in Figure 12.

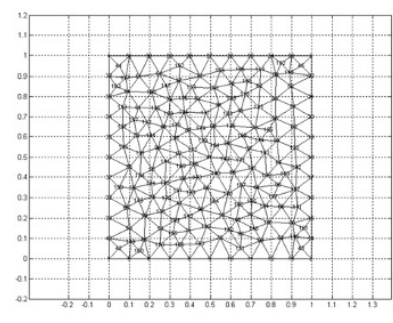

a)

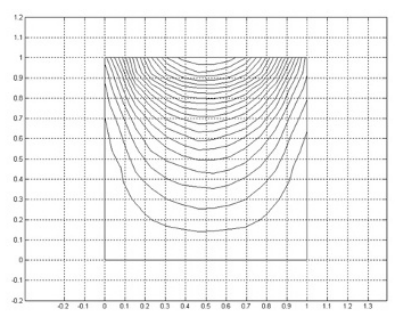

b)

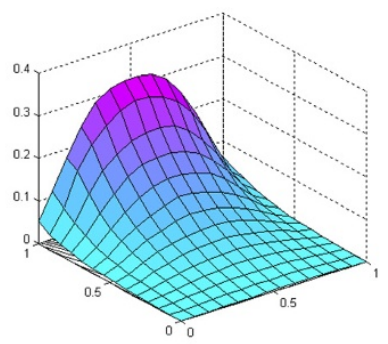

c)

Figure 11 Characteristics of a number of sensors related to an optimised solution of the partial derivative equation. a) Meshes and nodes $\mathbf{b})$ temperature isotherms, and $\mathbf{c}$ ) temperature in 3D. 


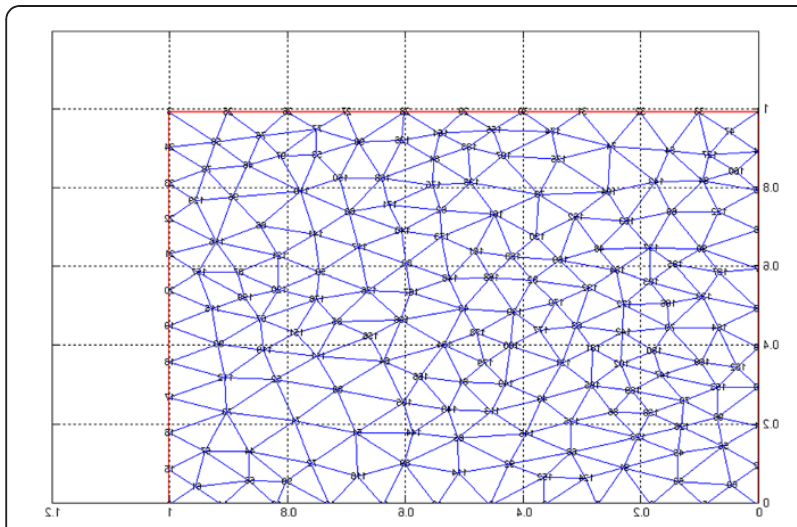

Figure 12 The optimised solution for meshes and nodes.

But in the case in which we have only a reduced number of sensors, for example only 13 sensors, the placement of these sensors in plane is presented in Figure 13.

In the case study a small sensor network with only 13 nodes was used in laboratory tests. The number of sensors is equivalent to a reduced number of nodes and meshes, like in the position scheme from Figure 13. In the case study we are choosing the nodes $8,13,12,5$ and 11 to apply the estimation method. These nodes are marked with bold characters in Figure 13. The transient characteristics of the temperature (in relative values) are presented in Figure 14, for 101 samples. The transient characteristics of the $12^{\text {th }}$ and $13^{\text {th }}$ nodes are the same, so they are plotted one over the other, and in Figure 14 there are only four characteristics instead of five.

An example of estimation for the $5^{\text {th }}$ node, based on auto-regression is presented as follows. The $5^{\text {th }}$ node is the node of the estimated variable, based on the autorecursive algorithm (13):

$$
\theta_{5}^{k+1}=f\left(\theta_{5}^{k}, \theta_{5}^{k-1}, \theta_{5}^{k-2}, \theta_{5}^{k-3}\right)
$$

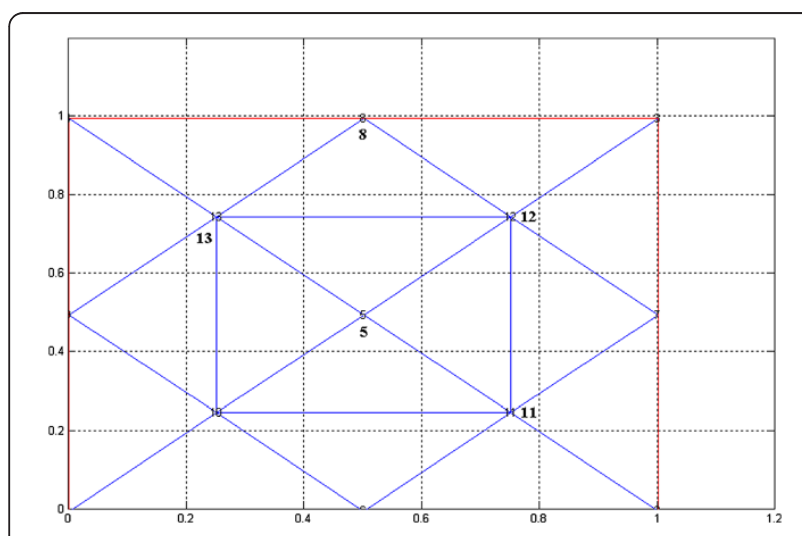

Figure 13 Sensor network position in the field.

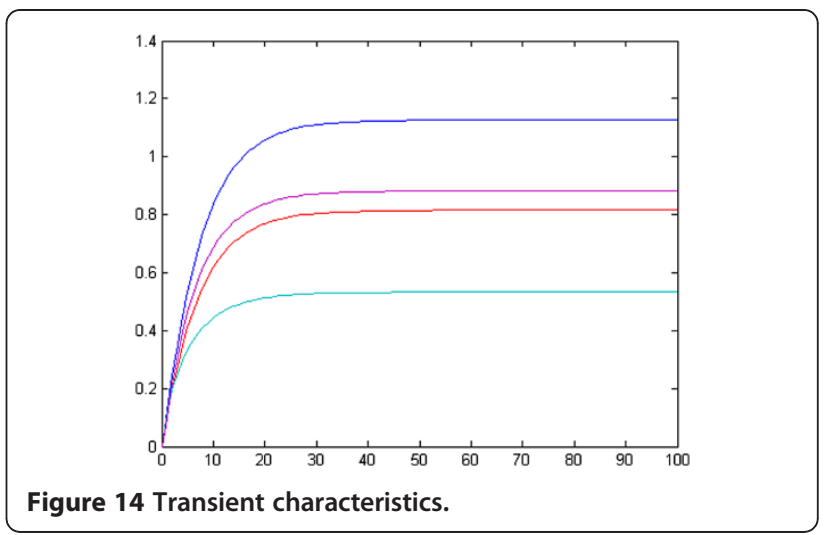

For the linear estimator an example of the coefficient values of a sensor in equation (14) is: $\mathrm{a}_{0}=1,2174 ; \mathrm{a}_{1}=$ $-0,2396 ; \mathrm{a} 2=0,1478 ; \mathrm{a}_{3}=-0,1261$.

An example of training error for the neural estimator with the neural structure from Figure 6 is presented in Figure 15.

The error is decreasing after 300 training epoch using the Levenberg-Marquardt method.

The comparison transient characteristics for training and testing output data for the estimator based on ANFIS are presented in Figure 16.

The characteristics are plotted two on the same graph, to show that there is no significant difference. The characteristic of the training data is plotted with ${ }^{\circ}$. The characteristic of the FIS output is plotted with *. The difference between the training case and the testing case is very small. The plotting signs ${ }^{\circ}$ and ${ }^{*}$ are on the same points for both characteristics. The average testing error is $2,017 \cdot 10^{-5}$. The number of training epochs was 3 .

If a fault appears in sensor 5, for example at the time moment of the 50th sample, an error occurs in estimation, like in Figure 17.

Detection of this error is equivalent to a default in sensor 5, from another point of view, in the place of sensor 5 , in the space of the distributed parameter systems, and in the heat flow around sensor 5.

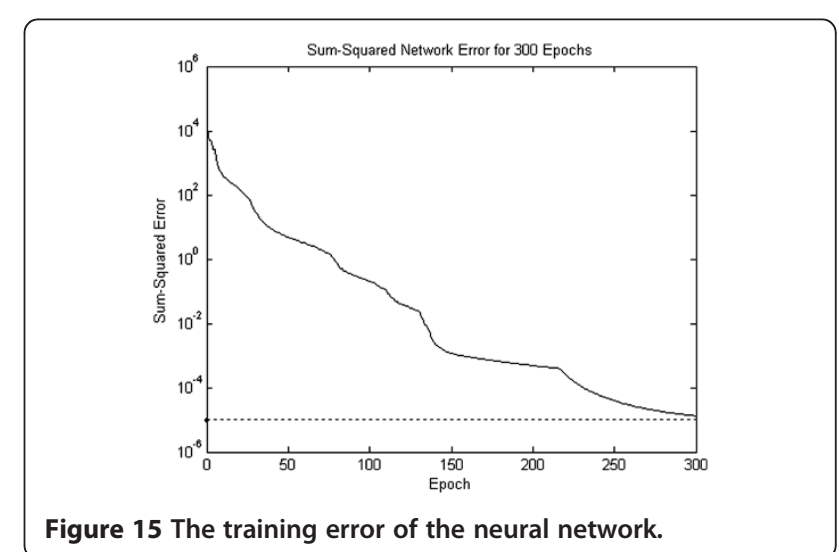




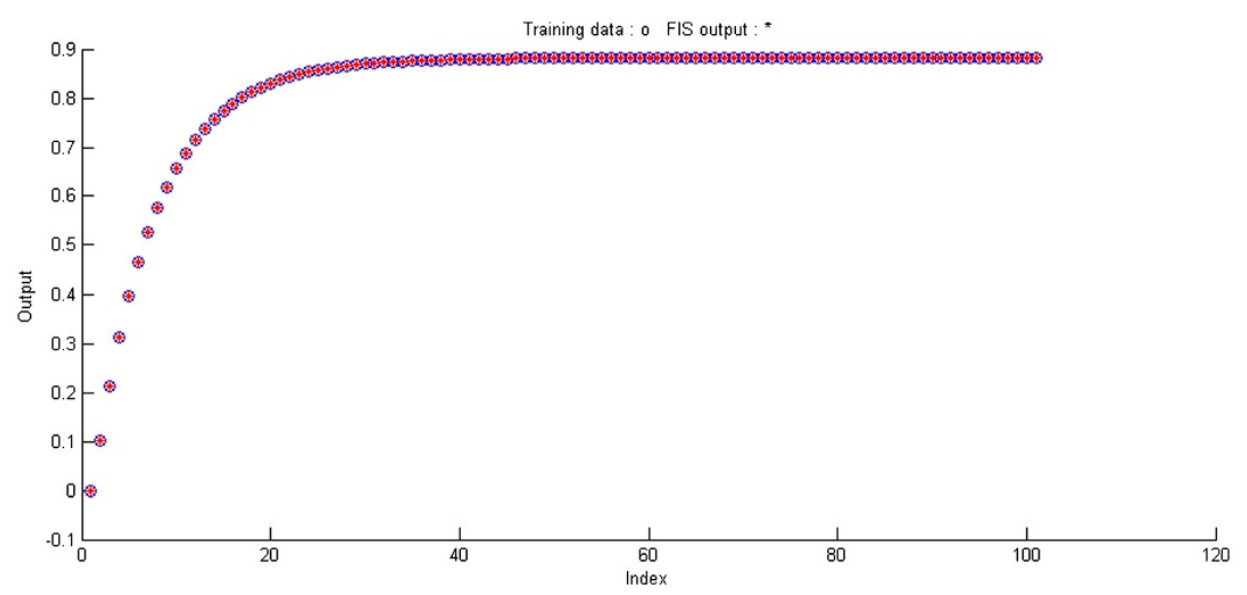

Figure 16 Measured and estimated transient characteristics.

\subsection{Comparison results}

After the comparison of the results of estimation obtained using these three methods the values of the quality criterion are synthesised in the following table.

In Table $1 e_{\mathrm{m}}$ is the average error, $e_{\mathrm{m}} \%$ is the error in percentage terms related to the measured values and $J$ is the quadratic criterion value obtained for the estimates. We may notice that the quality of estimation increases in Table 1 from top to bottom.

\section{Implementation}

A Crossbow sensor network was used in practice (Figure 18).

The basic components of the sensor network used in practice are a base station and sensor nodes. The base station is wireless, with computing energy and communication resources, which is acting like an access gate between the sensor nodes and the end user. The base station is an IRIS module, a gateway MIB250, which is connected at the USB. The senor nodes have a processor/radio module IRIS, which are activating

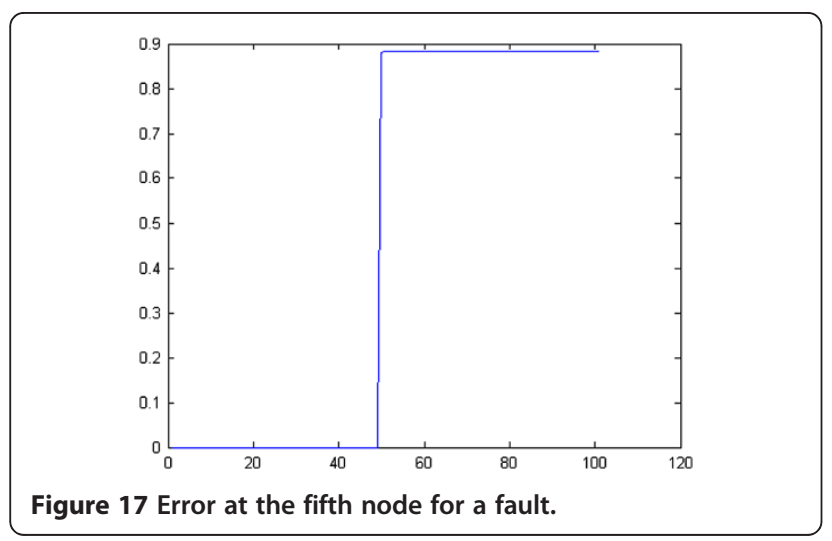

the measuring system of small power. They are working at the frequency of 2,4 GHz. The sensor circuit MTS400 includes a temperature sensor. The sensor network has also the software MoteView, for data acquisition, which is reading data from a database PostrgreSQL (Figure 19).

Virtual instrumentation, based on National Instruments technology, was used for sensor network monitoring. A virtual instrument was built on a personal computer, which includes: data acquisition and processing, estimator, data base, results table and an Excel data base. The monitoring system is working in real time. The sampling period is $9 \mathrm{~s}$. The control panel graphs for the measured and estimated temperature are presented in Figure 20a). The control panel graph for the estimation error is presented in Figure 20b).

The block diagram of the virtual instruments for sensor network monitoring is presented in Figure 21.

The block diagram is built on five levels of sub-VIs, including input-output virtual instruments and estimation sub-VIs. The coefficients of the virtual instrument are those obtained by off-line estimation. The driver assures data manipulation with a very small delay. The values obtained at long distance through the Internet are presented in Figure 22.

The data are sent at long distance, on the Internet, through an ethernet connection, being visualised by a browser.

Table 1 Quality results

\begin{tabular}{llll}
\hline Method & $\boldsymbol{e}_{\mathbf{m}}$ & $\mathbf{e}_{\mathbf{m}} \%$ & $\boldsymbol{J}$ \\
\hline Linear & 0,064 & 0,35 & 0,581 \\
Neural & 0,031 & 0,17 & 0,136 \\
ANFIS & 0,0258 & 0,14 & 0,0945 \\
\hline
\end{tabular}




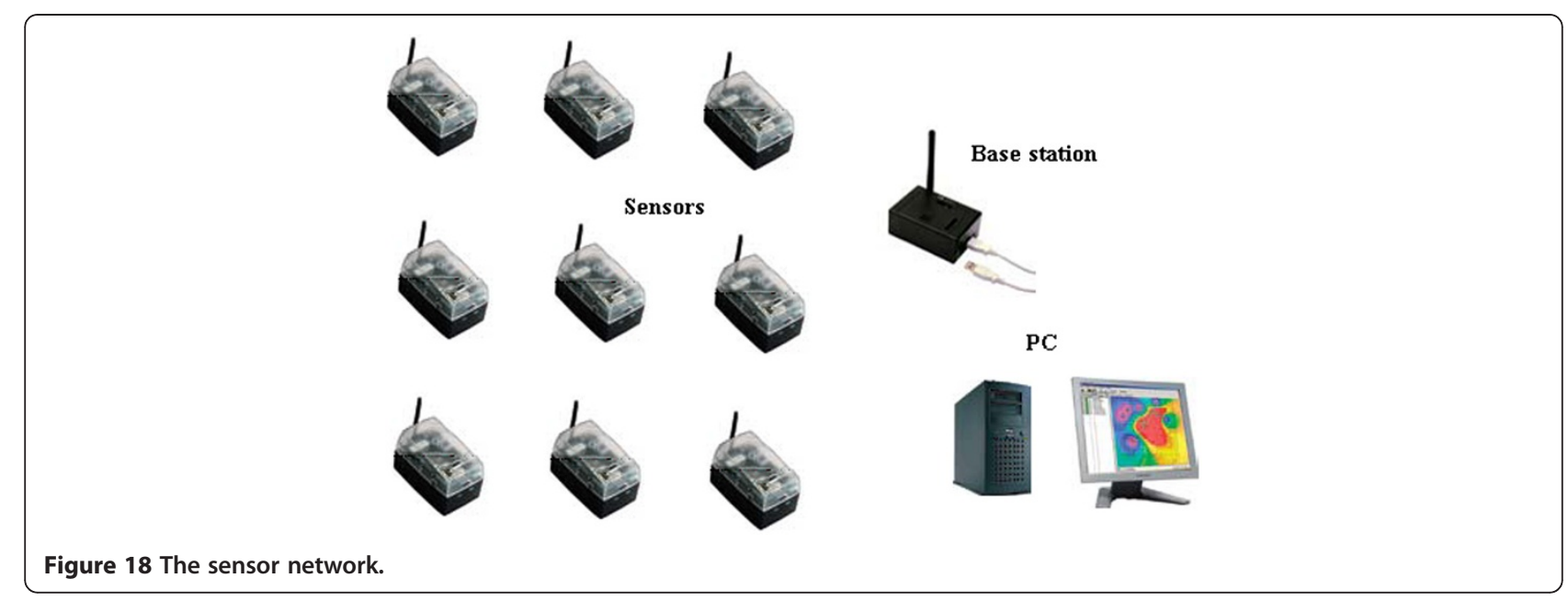

\section{Conclusions}

The paper has a technical contribution by implementing the scientific theory of distributed parameter systems with the linear and non-linear estimation techniques for a practical application using intelligent wireless sensor networks. This approach may be seen as a new technology based on specific estimation algorithms, using the sensor network as a "distributed" sensor place in the field of the distributed parameter systems, with application in the development of methods for monitoring, fault detection and diagnosis, and sensor malfunction detection.

The main contribution of this paper is a complex estimation structure, combining the two estimation algorithms, with regression and auto-regression, tested separately on other case studies, implemented using three efficient estimation methods: one linear and two non-linear - with neural networks and ANFIS. This complex system is used to estimate the value of the sensor at the moment $t+1$, based on the past values of the same sensor and also based on the current values of the adjacent sensors. The paper presents an implementation on a real sensor network of the complex estimator, using three existing fault detection and diagnosis algorithms. The complex estimator uses the efficiency of the linear and non-linear implementation methods.

The identification algorithms are in accord with the distributed parameter systems. The step between the considered mathematical models and the estimation

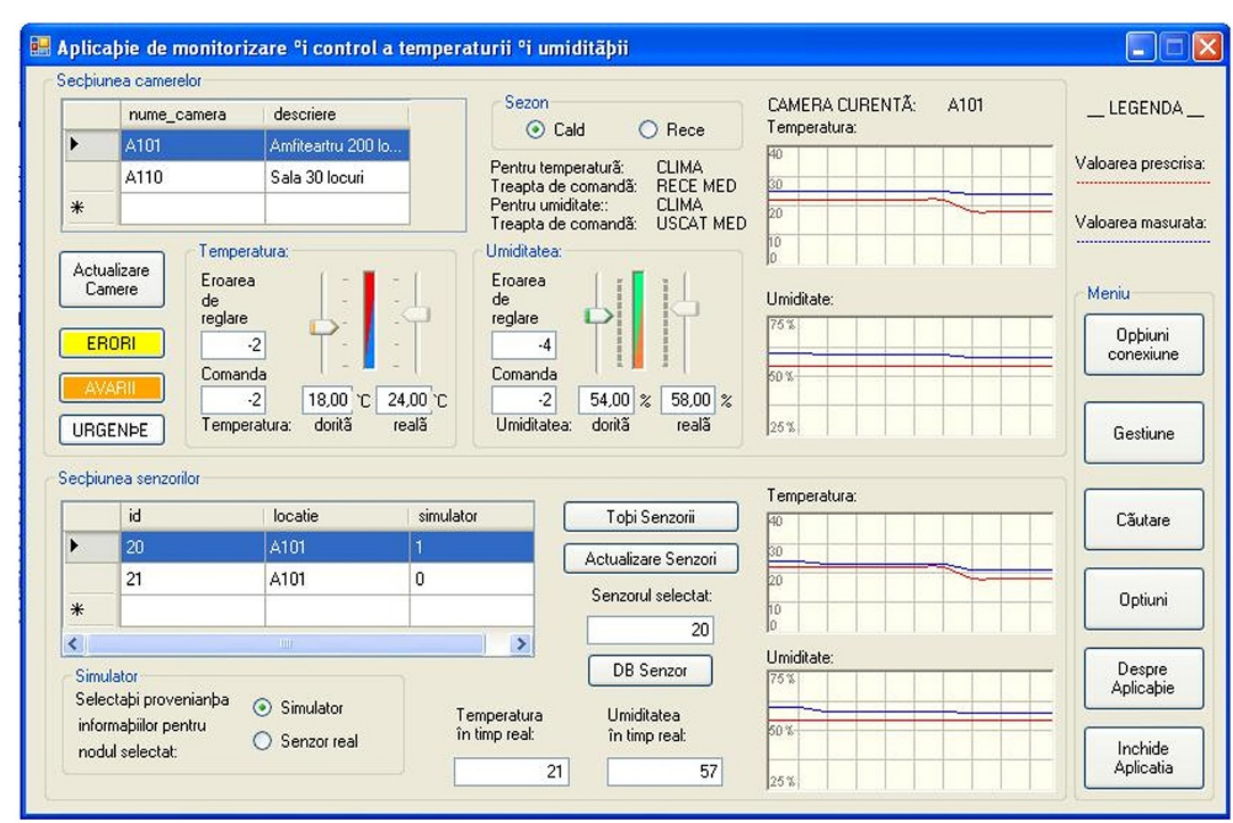

Figure 19 The user interface. 


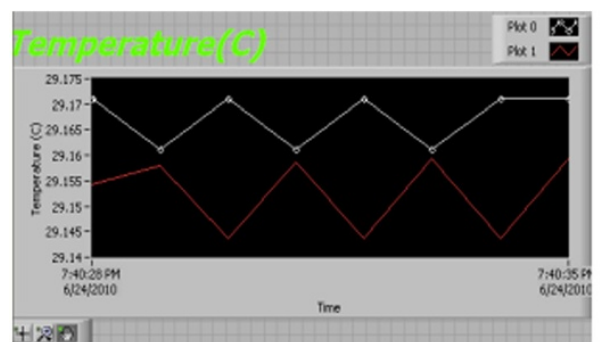

a)

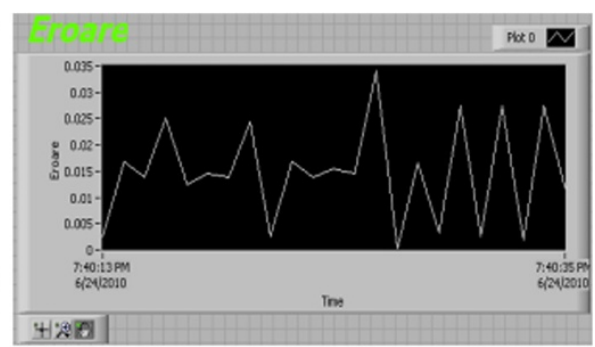

b)

Figure 20 Measurements on the control panel of the virtual instrument a) The measured and estimated temperature b) The error, on the control panel of the virtual instrument.

algorithms is obvious analyzing the form of the estimation algorithm and the general equation of a distributed parameter system.

The aspects arising in the identification of distributed parameter systems are analysed. The structure determination, variable selection and distributed computation are solved in practice.

The specific model structure imposed by the distributed parameter system dynamics is integrated into the estimation algorithms. The algorithms are designed based on knowledge obtained from the process seen as a distributed parameter system. The number of inputs is in accord with the process dynamics. The data acquired using sensor networks are filtered from the associated constraints of communication, computation and others specific to the technology of wireless sensor networks.

The sensor network has a major role in this approach, the development being done according to the modern facilities and capabilities of such an intelligent concept.

The main points of the paper may be summarised as follows. Some estimation algorithms, based on regression and auto-regression, for parabolic distributed parameters systems, are developed. They may be implemented using different linear or non-linear estimation methods - such as neural networks or ANFIS. A monitoring method based on a sensor network and these estimation algorithms is presented. A case study for the heat transfer in plane is presented. The results of a

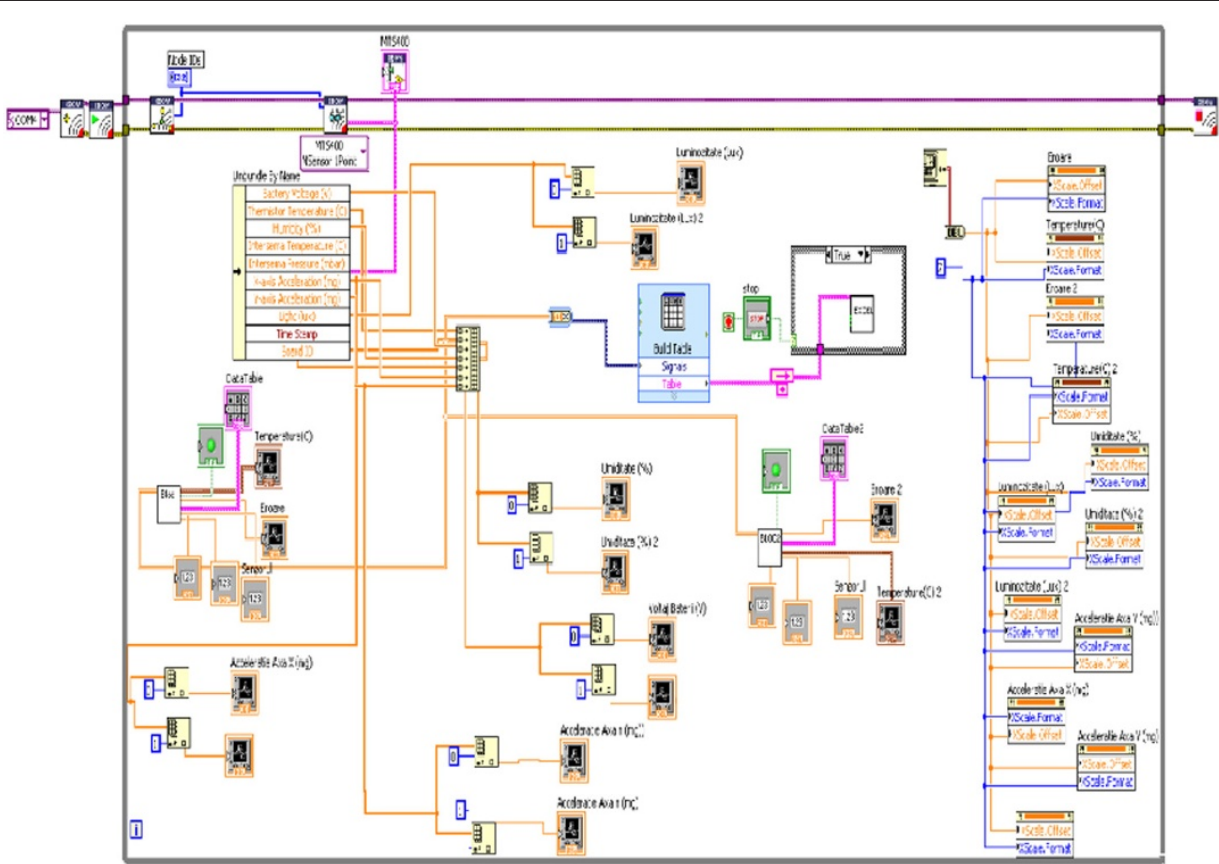

Figure 21 The block diagram of the virtual instrument. 


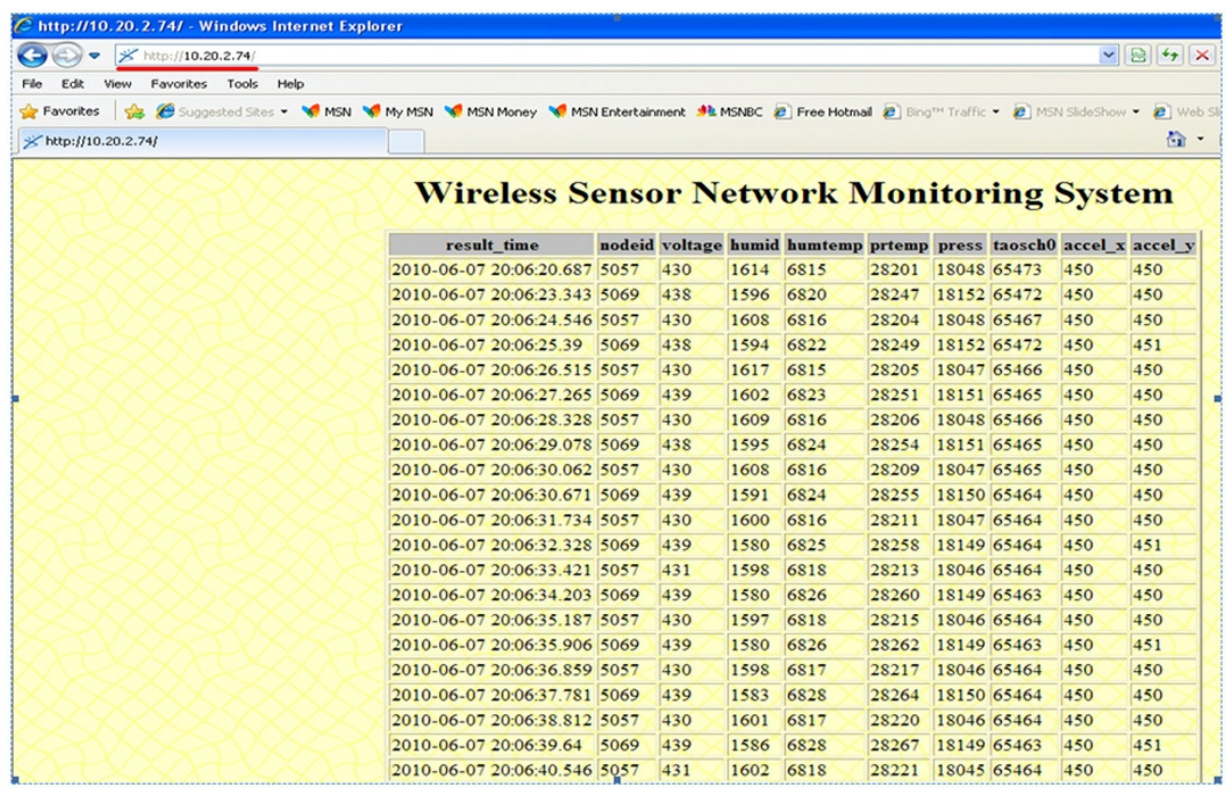

Figure 22 Data on Internet browser.

comparison study for these three methods, based on a quadratic quality criterion, are presented. Good approximations were obtained for all three methods. To improve the quality of the estimation one of these methods could be chosen, related to the desired error and the complexity of the implementation.

A virtual instrument was developed for the sensor network monitoring, including an estimator, based on the estimator coefficients obtained in an off-line estimator design.

A long distance monitoring over the Internet is developed.

The importance of the work consists in developing new estimator methods and implementation in distributed parameter systems. The main attention is given to the way in which to choose the number and the positions of the sensor nodes according to the desired accuracy in the identification process. Some examples of generated meshes and temperature estimates for different numbers of sensor are presented. A comparative study of how to place the sensor nodes in the field is made. A solution to know the right number of needed sensor nodes is offered. The users could know the right points in space to place the sensor nodes to obtain a good approximation by identification. The placing points are more important than the number of sensors.

A monitoring method based on a sensor network and ANFIS as estimation method for non-linear systems is presented.

The complex estimation system, based on the above estimation methods and algorithms, was developed to be implemented using virtual instrumentation.
Using this complex system the quality of estimation was improved, fact demonstrated by the good values obtained for the quadratic quality criteria.

The following applications and extensions are suggested: applications in fault detection and diagnosis of distributed parameter systems based on sensor networks and virtual instrumentation, allowing the treatment of large and complex systems with many variables, and also on learning and extrapolation. The monitoring method may also be applied in the case of discovery of malicious nodes in wireless sensor networks. New applications may be developed in the future, considering all the capabilities of the sensor nodes to measure physical variables.

\section{Competing interests}

The authors declare that they have no competing interests.

\section{Acknowledgement}

This work was developed within the frame of PNII-IDEI-PCE-ID923-2009 CNCSIS - UEFISCSU grant.

Received: 5 December 2011 Accepted: 3 December 2012

Published: 9 January 2013

\section{References}

1. C. Volosencu, Algorithms for estimation in distributed parameter systems based on sensor networks and ANFIS. WSEAS Transactions on Systems 9(3), 283-294 (2010)

2. C. Volosencu, Identification of distributed parameter systems, based on sensor networks and artificial intelligence. WSEAS Transactions on Systems 7 (6), 785-801 (2008). ISSN 1109-2777

3. C. Volosencu, D.I. Curiac, Monitoring of distributed parameter systems based on a sensor network and ANFIS, in 2010 IEEE World Congress on Computational Intelligence (IJCNN volume, Barcelona, Spain, 2010), pp. 2272-2279

4. C. Volosencu, Applying the technology of wireless sensor network in environment monitoring, in Cutting Edge Research in New Technologies, ed. by C. Volosencu (Intech, Rijeka, 2012), pp. 97-106 
5. D.I. Curiac, C. Voloşencu, A. Doboli, O. Dranga, T. Bednarz, Discovery of malicious nodes in wireless sensor networks using neural predictors. WSEAS Transactions on Computer Research 2(1), 38-44 (2007). ISSN 1991-8755

6. I.F. Akyildiz, W. Su, Y. Sankarasubramaniam, E. Cayirci, Wireless senso networks: a survey. Computer Networks 38(4) (2002)

7. B. Krishnamachari, A Wireless Sensor Networks Bibliography (University of Southern California, Technical Report, 2007)

8. R.U. Novak, U. Mira, Boundary estimation in sensor networks: theory and methods, in 2nd International Workshop on Information Processing in Sensor Networks (IPSN, Palo Alto, CA, 2003)

9. G.J. Pottie, W.J. Kaiser, Wireless integrated network sensors. Communications of the ACM 43 (2000)

10. L. Tong, Q. Zhao, S. Adireddy, Sensor Networks with Mobile Agents, Proceedings IEEE 2003 MILCOM (, Boston, USA, 2003), pp. 688-694

11. H. Allende-Cid, A. Veloz, R. Salas, S. Chabert, H. Allende, Self Organizing Neuro-Fuzzy System, in Progress in Pattern Recognition (Image Analysis and Applications, Springer Verlag, Heidelberg, 2008)

12. G. Battistelli, A. Benavoli, L. Chisci, State Estimation with Remote Sensors and Data Driven Communication, Estimation and Control of Networked Systems, Volume 1, Part 1, 2010 (Artigianelli, Italy, 2010)

13. G. Biagetti, P. Crippa, F. Gianfelici, C. Turchetti, Sensor Network-Based Nonlinear System Identification, Lecture Notes in Computer Science (Springer Berlin, Heidelberg, ), pp. 580-587

14. M. Patan, D. Ucinski, Configuring a sensor network for fault detection in distributed parameter systems. Int J Appl Math Comput Sci 18(4), 513-524 (2008)

15. L. Rauchhaupt, V. Lakkundi, Wireless network integration into virtual automation networks, in Proceedings of the 17th IFAC World Congress, Volume 17, part 1 (, Seoul, South Korea, 2008), pp. 13982-13987

16. M. Tubaishat, S. Mandria, Sensor networks: an overview. IEEE Potential 22(2), 20-23 (2003)

17. L. Ljung, Perspectives on system identification. Annual Reviews in Control 34(1), 1-12 (2010). Elsevier

18. P. Morreale, R. Suleski, System design and analysis of a web-based application for sensor network data integration and real-time presentation, in Proceedings of the 3rd IEEE Systems Conference (, Vancouver, British Columbia, Canada, 2009), pp. 201-204

19. T.P. Lambrou, C.G. Panayiotou, Collaborative area monitoring using wireless sensor networks with stationary and mobile nodes. EURASIP Journal on Advances in Signal Processing (2009). doi:10.1155/2009/750657. vol. 2009, Article ID 750657, 16 pages

20. D. Dardari, C.C. Chong, B. Damien, D.B. Jourdan, L. Mucchi, Cooperative localization in wireless ad hoc and sensor networks. EURASIP Journal on Advances in Signal Processing (2008). doi:10.1155/2008/353289. vol. 2008, Article ID 353289, 2 pages, 2008

21. V.A. Kaseva, M. Kohvakka, M. Kuorilehto, M. Hännikäinen, T.D. Hämäläinen, A wireless sensor network for rf-based indoor localization. EURASIP Journal on Advances in Signal Processing (2008). doi:10.1155/2008/731835. vol. 2008, Article ID 731835, 27 pages

22. E. Serpedin, H. Li, A. Dogandžić, H. Dai, P. Cotae, Distributed signa processing techniques for wireless sensor networks. EURASIP Journal on Advances in Signal Processing (2008). doi:10.1155/2008/540176. vol. 2008, Article ID 540176, 2 pages

23. J.C. Chen, A.G. Jaffer, Editorial. EURASIP Journal on Applied Signal Processing 2005(1), 1-3 (2005). doi:10.1155/ASP.2005.1

24. K. Yao, D. Estrin, H.Y. Hu, Editorial. EURASIP Journal on Applied Signal Processing 2003(4), 319-320 (2003). doi:10.1155/S1110865703002944

25. I. Chatzigiannakis, G. Mylonas, S. Nikoletseas, The design of an environment for monitoring and controlling remote sensor networks. International Journal of Distributed Sensor Networks 5, 262-282 (2009)

26. S. Jassar, T. Behan, L. Zhao, Z. Liao, The comparison of neural network and hybrid neuro-fuzzy based inferential sensor models for space heating systems, in IEEE International Conference on Systems, Man and Cybernetics, SMC, 2009 (, San Antonio, Texas, 2009), pp. 4299-4303

27. Q. Yang, A. Lim, K. Casey, R.K. Neelisetti, An enhanced CPA algorithm for real-time target tracking in wireless sensor networks. International Journal of Distributed Sensor Networks 5, 619-643 (2009)

28. B. Shen, Z. Wang, Y.S. Hung, Distributed $\mathrm{H}_{\infty}$-consensus filtering in sensor networks with multiple missing measurements: The finite-horizon case. Automatica 46(10), 1682-1688 (2010)

29. S.H. Hsu, K.S. Chen, H.R. Lin, S.J. Chang, T.P. Tang, Effect of plasma gas flow direction on hydrophilicity of polymer by small zone cold plasma treatment and hydrophobic plasma treatment. International Journal of Distributed Sensor Networks 5, 429-436 (2009)

30. H. Zhang, J.M.F. Moura, B. Krogh, Estimation in sensor networks: a graph approach, in 4th International Symposium on Information Processing in Sensor Networks (, Los Angeles, CA, 2005), pp. 203-209

31. H. Zhang, B. Krogh, J.M.F. Moura, Estimation in virtual sensor-actuator arrays using reduced-order physical models, in CDC'04 43rd IEEE Conference on Decision and Control, vol. 4 (, Bermudas, 2004), pp. 3792-3797

32. M. Farina, G. Ferrai-Trecate, R. Scattolini, Estimation and Control of Networked Systems, Vol. 1, Part 1 (, Artigianelli, Italy, 2010)

33. G.C. Calafiore, F. Abrate, Distributed linear estimation over sensor networks Int Journal of Control 82(5), 1868-1882 (2009)

34. L. Shi, K.H. Johansson, M.R. Murray, Estimation Over Wireless Sensor Networks: Trade-off between Communication, Computation and Estimation Qualities, Proc. of the 17th IFAC World Congress (, Seoul, South Korea, 2008)

35. R. Saifan, O. Al-Jarrah, A novel algorithm for defending path-based denial of service attacks in sensor networks. International Journal of Distributed Sensor Networks (2010). doi:10.1155/2010/793981. Volume 2010, Article ID 793981

36. Y.F. Zhu, H.Z. Tan, P. Wan, Y. Zhang, A blind approach to non-linear system identification, in IET Conference on Wireless, Mobile and Sensor Networks, 2007 (CCWMSN07, Shanghai, China, 2009), pp. 209-212

37. I. Chairez, R. Fuentes, A. Poznyak, T. Poznyak, M. Escudero, L. Viana, Neural network identification of uncertain 2D partial differential equations, in 6th International Conference on Electrical Engineering, Computing Science and Automatic Control, CCE, 2009 (, Toluca, Mexico, 2009), pp. 1-6

38. A. Depari, A. Flammini, D. Marioli, A. Taroni, Application of an ANFIS algorithm to sensor data processing. IEEE Trans on Instrumentation and Measurement 56(1), 75-79 (2005)

39. J. Yue, J. Liu, X. Liu, W. Tan, Identification of non-linear system based on ANFIS with subtractive clustering, in The Sixth World Congress on Intelligent Control and Automation, WCICA 2006, vol. 1 (, Dalian, China, 2006), pp. 1852-1856

40. C. De-Wang, Z. Jun-Ping, Time series prediction based on ensemble ANFIS, in Proceedings of the 2005 International Conference on Machine Learning and Cybernetics, vol. 6 (, Guangzhou, China, 2005), pp. 3552-3556

41. E. Franco, R. Olfati-Saber, T. Parisini, M.M. Polycarpou, Distributed fault diagnosis using sensor networks and consensus-based filters, in 45th IEEE Conference on Decision and Control (, San Diego, CA, 2006), pp. 386-391

doi:10.1186/1687-6180-2013-4

Cite this article as: Volosencu and Curiac: Efficiency improvement in multi-sensor wireless network based estimation algorithms for distributed parameter systems with application at the heat transfer. EURASIP Journal on Advances in Signal Processing 2013 2013:4.

\section{Submit your manuscript to a SpringerOpen ${ }^{\odot}$ journal and benefit from:}

- Convenient online submission

- Rigorous peer review

- Immediate publication on acceptance

Open access: articles freely available online

- High visibility within the field

- Retaining the copyright to your article

Submit your next manuscript at springeropen.com 